\title{
BEAM BENDING PROBLEMS ON A PASTERNAK FOUNDATION USING RECIPROCAL VARIATIONAL INEQUALITIES*
}

\author{
BY \\ NOBORU KIKUCHI \\ The University of Texas at Austin
}

\begin{abstract}
The present study is concerned with a class of two-body contact problems in linear elasticity. The model problem is a bending problem of the beam resting unilaterally upon a Pasternak foundation. A variational formulation is given by the minimax principle of the functional, and proof of the existence of saddle points is given by the compatibility condition for applied forces and moments on the beam. It has been found that the compatibility condition for equilibrium of the beam and foundation can be achieved by arguments of coerciveness of the functional on the admissible set. An approximation and example of the problem by finite element methods and a numerical method for its solution are also introduced.
\end{abstract}

1. Introduction. The present study is concerned with a class of two-body contact problems in linear elasticity. More specifically, a bending problem for a beam which is unilaterally resting on a Pasternak foundation without support or fixed conditions is considered by the theory of convex analysis. In this paper, a mathematical theory for such a problem as well as a method for obtaining an approximate solution are introduced.

The contact problem is formulated by deflection of the beam and contact pressure. One advantage of using contact pressure is that the problem can be defined on the domain of the beam even though the foundation is infinitely long. Moreover, constraint inequalities due to unilateral contact are given by contact pressure, but not by deflection of the beam. This makes it simple to formulate the problem. However, it should be pointed out that concentrated contact forces may occur at the end points of the beam when they contact the foundation due to the characteristics of the Pasternak foundation. Indeed, since the Pasternak foundation is represented by the second-order differential equation of the deflection, the first derivative of its deflection need not be continuous, whereas the first derivative of the deflection of the beam is always continuous. If contact between the beam and the foundation occurs within the interior of the beam, the first derivative of deflection of the foundation is then forced to maintain continuity; that is, contact pressure is distributed. If, on the other hand, the beam contacts the foundation at its end points, the first derivative of the deflection of the foundation does not have to be continuous. Discontinuitv implies concentrated force at the end noints.

* Received March 14, 1979; revised version received July 11, 1979. The author was supported during this study by the Air Force Office of Scientific Research grant F-49620-78-C-0083. 
For the formulation of the contact problem which is discussed in this paper, a variational principle is introduced using the minimax principle of the functional. The functional consists of the total potential energy of the beam and the reciprocal potential energy of the foundation. Here reciprocal potential energy means total potential energy and is expressed by the force instead of the deflection.

Next, existence of a saddle point of the functional associated with the contact problem is proved. To this end, precise definitions of the admissible sets for deflections of the beam and contact pressure of the foundation are provided. The admissible set of deflection of the beam is chosen such that the total strain energy of the beam is finite. This in turn restricts the possibility of applied forces, moments, etc.; that is, the work done by any external forces and moments must be a continuous linear functional on the admissible set of deflections of the beam. The admissible set of contact pressure is chosen as a subset of the dual of the space in which the strain energy of the Pasternak foundation is finite. Because of unilateral contact, admissible contact pressures are restricted by the inequality $\leq$ which is an extended relation of $\leq$ in the ordinary sense to the dual space.

Thus, once the admissible sets are defined, existence of a saddle point is proved. A sufficient condition for the existence of a saddle point is obtained by arguing the coerciveness of the functional. Even though the beam is not supported or fixed, a saddle point (i.e. an equilibrium configuration of the beam and foundation) exists if applied forces and moments satisfy the compatibility condition. This condition can be obtained only through rigorous theoretical arguments.

An approximation of the saddle point problem, which represents the contact problem, is next introduced. For the beam, the first-order Hermite interpolation is taken in each finite element of the beam. For the foundation, the contact pressure is interpolated by the linear combination of the Dirac delta functions. The saddle point problem then becomes a system of equations with respect to the generalized displacement of the beam, and a system of inequalities with respect to the contact pressure of the foundation. The system of equations is treated as usual. A solution method for the system of inequalities is developed through consideration of a simple one-variable problem in which characteristics are equivalent to those associated with the present problem. The solution method introduced here is based on Uzawa's iterative method which is discussed in Ekeland and Temam [1].

In considering the present problem, the works of Fremond [2] and Boucher [3] were particularly relevant. Fremond solves an indentation problem between a linearly elastic body and a semi-infinite linearly elastic foundation using the theory of variational inequalities. He represents the infinite domain by the Green function which is obtained using the so-called Kelvin solution, e.g., Fung [4]. This procedure is also applied in the present study because representation of the foundation by contact pressure provides a more direct relationship between the body and the foundation. However, in this paper, the unilateral contact condition is imposed only on the pressure and not on the deformation of the beam. Contact constraint is represented by both the deformation of the body and the pressure of the foundation in Fremond [2]. Because the constraint is imposed only on the contact pressure, the present formulation can be much simpler than that of Fremond. Boucher [3] took another approach to the same problem. He defined the contact problem by deformations of both the indented body and the foundation instead of by contact pressure. He also found a sufficient condition for the existence of 
solutions in the indented body that has no fixed conditions. A similar procedure is adopted to find a sufficient condition in the present problem since the beam is not supported or fixed at any point.

The general mathematical background of this study is provided by Ekeland and Temam [1] and Nečas [5]. The minimax principle of the functional and Uzawa's iteration method are extracted from Ekeland and Temam [1], and consequences of function spaces, such as the Sobolev space, are found in Nečas [5].

2. A beam bending problem. Suppose that a beam resting upon a Pasternak foundation is subjected to the transverse load $f(x)$ applied within the interior of the beam and to the axial load $P$ applied at the end points of the beam. Suppose that the beam rests on the foundation unilaterally with no fixed boundary conditions. If some part of the beam contacts the foundation, contact pressure occurs. On the other hand, wherever the beam leaves the foundation, the contact pressure becomes zero. In this section governing equations are derived using both the deflection of the beam and the contact pressure of the foundation.

Let the beam occupy the interval $(0, l)$ of the $x$-axis initially, and let it be characterized by the linear differential equation

$$
\left(E I w^{\prime \prime}\right)^{\prime \prime}-P w^{\prime \prime}=\hat{f} \text { in }(0, l) .
$$

Here $E I$ is the stiffness of the beam, $\hat{f}$ is the net transverse load, $P$ is the applied axial load, and the prime indicates the derivative with respect to $x$. If $P$ is positive, the beam receives tension force.

Let the foundation occupy the infinite region $(-\infty, \infty)$ of the $x$-axis. The Pasternak foundation is characterized by the linear differential equation

$$
-\left(\mu u^{\prime}\right)^{\prime}+k u=\hat{p} \text { in }(-\infty, \infty),
$$

where $\mu$ is the shear modulus, $k$ is the spring constant, $\hat{p}$ is the net transverse load (pressure), and $u$ is the deflection of the foundation. The Pasternak foundation is a simple model of the real foundation consisting of a membrane and continuously distributed springs. If $\mu=0$, the Pasternak foundation coincides with the Winker foundation. Details of such simple foundation models can be found in Keer [6].

By using material constants $\mu$ and $k$ of the Pasternak foundation, the solution of the differential equation (2.2) is given by the integral form

$$
u(x)=\int_{-\infty}^{\infty} g(x, y) \hat{p}(y) d y=G(\hat{p})(x)
$$

where

$$
g(x, y)=\frac{1}{2 \mu b} \mid e^{b(x-y)} \quad \text { if } \quad x<y
$$

and

$$
b^{2}=k / \mu .
$$

The contact problem, shown in Fig. 1, is next formulated. First, properties of contact pressure are investigated. If contact occurs within the beam, only distributed contact pressure $p(x), 0<x<l$, is produced because the gradient of the deflection of the beam is 


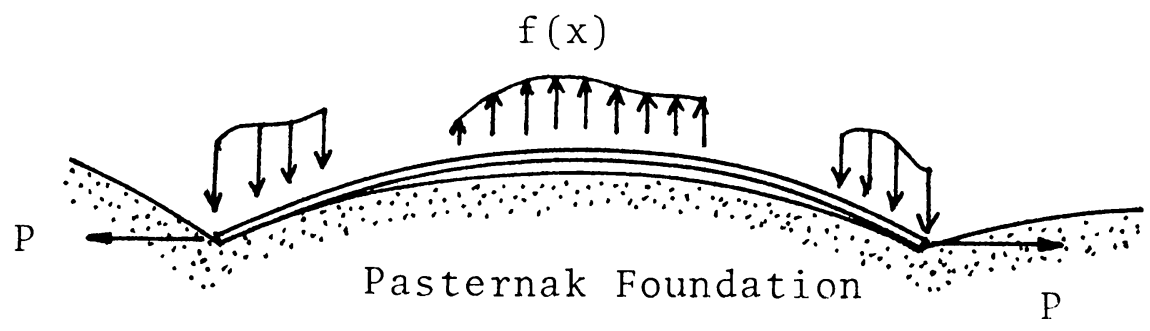

FIG. 1. Beam on the Pasternak foundation.

continuous in the whole region $(0, l)$. However, if the end points of the beam contact the foundation, the gradient of the deflection of the Pasternak foundation is no longer continuous since the gradient of the beam deflection is not defined at the end points. This causes concentrated reacting forces at the end points of the beam, say $p_{0}$ and $p_{l}$. Thus, the contact pressure $\hat{p}$ consists of three components $\left\{p(x), p_{0}, p_{l}\right\}$; more precisely,

$$
\begin{array}{rlrl}
\hat{p}(x) & =0, & & -\infty<x<0 \\
& =p_{0}, & & x=0 \\
& =p(x), & & 0<x<l \\
& =p_{l}, & & x=l \\
& =0, & l<x<+\infty,
\end{array}
$$

Furthermore, since the beam contacts the foundation only unilaterally, the reacting contact pressure $\hat{p}(x)$ cannot take negative values on the entire domain $(-\infty, \infty)$, i.e.,

$$
\begin{array}{lll}
\hat{p}(x)=0 & \text { if } \quad u(x)>w(x) \\
\hat{p}(x) \geq 0 & \text { if } \quad u(x)=w(x)
\end{array} \quad \text { on } \quad[0, l]
$$

and

$$
\hat{p}(x)=0 \quad \text { in }(-\infty, 0) \cup(l,+\infty) .
$$

The kinematical restriction due to contact is given by

$$
u(x) \geq w(x) \text { on }[0, l] .
$$

Here $[0, l]$ means the closed interval of the $x$-axis. Combining (2.7) through (2.9) yields the compact form of the contact conditions

$$
\begin{aligned}
& \hat{p}(x)(u(x)-w(x))=0, \quad \hat{p}(x) \geq 0, \quad u(x)-w(x) \geq 0 \quad \text { in }[0, l], \\
& \hat{p}(x)=0 \quad \text { in } \quad(-\infty, 0) \cup(l,+\infty) .
\end{aligned}
$$

On the other hand, the equilibrium equations of the beam are now represented by

$$
\begin{aligned}
\left(E I w^{\prime \prime}\right)^{\prime \prime}-P w^{\prime \prime}+p & =f \text { in }(0, l), \\
E I w^{\prime \prime}(0) & =E I w^{\prime \prime}(l)=0, \\
\left(E I w^{\prime \prime}\right)^{\prime}(0)+p_{0} & =-\left(E I w^{\prime \prime}\right)^{\prime}(l)+p_{l}=0 .
\end{aligned}
$$

The derivation of Eq. (2.11) from (2.1) is obvious since the net transverse load $\hat{f}(x)$ is 
equal to $f(x)-p(x)$, where $f(x)$ is the load applied to the beam. Since both of the end points of the beam are free, the bending moment must vanish, i.e., (2.12). The balance of shear forces at the end points of the beam is given by (2.13).

Thus, the unilateral contact problem described in Fig. 1 is formulated in a pointwise manner by the set of equations and inequalities $\{(2.3),(2.10),(2.11),(2.12)$, and $(2.13)\}$. In these representations, pointwise existence of $w^{\prime \prime},\left(E I w^{\prime \prime}\right)^{\prime \prime}$, and $p$ is assumed a priori. These requirements are weakened by variational formulation, as shown in the next section.

3. Variational formulation. In this section, a variational formulation associated with the contact problem discussed in the previous section is introduced. The variational formulation is established by the use of saddle-point methods of convex-concave functionals. To this end, the total potential energy of the Pasternak foundation due to the contact pressure is first derived. Let $\hat{q}(x)$ be an arbitrary contact pressure defined as in (2.6) such that $\hat{q}(x) \geq 0$. Then the total potential energy is defined by

$$
\begin{aligned}
F_{R}(\hat{q}) & =\frac{1}{2}\langle\hat{q}, G(\hat{q})\rangle-\langle\hat{q}, \hat{v}\rangle \\
& =\frac{1}{2} \int_{-\infty}^{\infty} \int_{-\infty}^{\infty} g(x, y) \hat{q}(y) d y \hat{q}(x) d x-\int_{-\infty}^{\infty} \hat{v}(x) \hat{q}(x) d x
\end{aligned}
$$

where $\hat{v}(x)$ is the extension of an arbitrary virtual deflection of the beam into the whole region $(-\infty, \infty)$ so that

$$
\begin{aligned}
\hat{v}(x) & =v(x), & & x \in[0, l] \\
& =0, & & x \in(-\infty, 0) \cup(l,+\infty) .
\end{aligned}
$$

The following identity follows from the fact that $\hat{q}(x)=0$ in $(-\infty, 0) \cup(l,+\infty)$ :

$$
\begin{aligned}
G(\hat{q})(x) & =\int_{-\infty}^{\infty} g(x, y) \hat{q}(y) d y \\
& =\int_{0}^{l} q(x, y) q(y) d y+g(x, 0) q_{0}+g(x, l) q_{l} .
\end{aligned}
$$

Then, using the symmetry $g(x, y)=g(y, x)$,

$$
\langle\hat{q}, G(\hat{p})\rangle=\langle\hat{p}, G(\hat{q})\rangle
$$

and

$$
\langle\hat{q}, G(\hat{p})\rangle=\int_{0}^{l} G(\hat{p})(x) q(x) d x+G(\hat{p})(0) q_{0}+G(\hat{p})(l) q_{l} .
$$

For the pairing $\langle\hat{q}, \hat{v}\rangle$, the following identity is valid:

$$
\langle\hat{q}, \hat{v}\rangle=\int_{0}^{l} v(x) q(x) d x+v(0) q_{0}+v(l) q_{l} .
$$

Therefore,

$$
\lim _{\theta \rightarrow 0} \frac{1}{\theta}\left[\frac{1}{2}\langle\hat{p}+\theta \hat{q}, G(\hat{p}+\theta \hat{q})\rangle-\langle\hat{p}+\theta \hat{q}, \hat{v}\rangle\right]=\langle\hat{q}, G(\hat{p})-\hat{v}\rangle
$$


The potential energy of the beam is written by

$$
F_{w}(v)=\frac{1}{2} \int_{0}^{l}\left\{E I\left(v^{\prime \prime}\right)^{2}+P\left(v^{\prime}\right)^{2}-2 f v\right\} d x .
$$

Thus, the Lagrangian $L(v, \hat{q})$ is defined by

$$
L(v, \hat{q})=\frac{1}{2} \int_{0}^{l}\left\{E I\left(v^{\prime \prime}\right)^{2}+P\left(v^{\prime}\right)^{2}-2 f v\right\} d x-\frac{1}{2}\langle\hat{q}, G(\hat{q})\rangle+\langle\hat{q}, \hat{v}\rangle
$$

where $\hat{v}$ is the extension of $v$, cf. (3.2). Let $V$ be the set of all admissible deflections of the beam, and let $K$ be the set of all admissible contact pressures of the foundation such that they are non-negative. Precise definitions of $V$ and $K$ will be given later.

Suppose that the pair $\{w, \hat{p}\} \in V \times K$ forms a saddle point of $L(\cdot, \cdot)$ on $V \times K$, i.e.,

$$
L(w, \hat{q}) \leq L(w, \hat{p}) \leq L(v, \hat{p}), \quad \forall(v, \hat{q}) \in V \times K .
$$

It will be shown that the saddle point $\{w, \hat{p}\}$ weakly satisfies the contact problem $(2.10)$ through (2.13).

Taking $\hat{q}=\hat{p}+\theta(\hat{s}-\hat{p}), \theta \in(0,1]$ and $\hat{s} \geq 0$ in the first inequality of (3.10) yields

$$
\frac{1}{2}\langle\hat{p}+\theta(\hat{s}-\hat{p}), G(\hat{p}+\theta(\hat{s}-\hat{p}))\rangle-\frac{1}{2}\langle\hat{p}, G(\hat{p})\rangle-\theta\langle\hat{s}-\hat{p}, \hat{w}\rangle \geq 0 .
$$

Dividing by $\theta>0$, and passing to the limit $\theta \rightarrow 0$ imply that

$$
\langle\hat{s}-\hat{p}, G(\hat{p})-\hat{w}\rangle \geq 0, \quad \forall \hat{s} \geq 0 .
$$

If $\hat{s}=\hat{p}+\hat{q}, \hat{q} \geq 0$, in (3.11), $\langle\hat{q}, G(\hat{p})-\hat{w}\rangle \geq 0$, i.e.

$$
\int_{0}^{l}(G(\hat{p})-w)(x) q(x) d x+(G(\hat{p})-w)(0) q_{0}+(G(\hat{p})-w)(l) q_{l} \geq 0 .
$$

Since the choice of $\hat{q}$ is arbitrary within non-negativeness, the inequality means that

$$
G(\hat{p})(x)-w(x) \geq 0 \text { in }[0, l] .
$$

According to (2.3), this is the same as the inequality

$$
u(x)-w(x) \geq 0 \text { in }[0, l] .
$$

Furthermore, if $\hat{s}=0$ and $\hat{s}=2 \hat{p}$ are taken in (3.11), it can be established that

$$
\langle\hat{p}, G(\hat{p})-\hat{w}\rangle=0 .
$$

Then the relation

$$
(G(\hat{p})-w)(x) \hat{p}(x)=0 \quad \text { in } \quad[0, l]
$$

follows from the definition (3.5) of $\langle\cdot, \cdot\rangle$. Since $\hat{p}(x) \in K$, the saddle point $\{w, \hat{p}\}$ satisfies

$$
(G(\hat{p})-w)(x) \hat{p}(x)=0, \quad \hat{p}(x) \geq 0, \quad u(x)-w(x) \geq 0 \quad \text { in } \quad[0, l] .
$$

These are exactly the same as the contact conditions (2.10) discussed in the previous section.

By taking $v=w \pm \theta z$ in the second inequality of (3.10), dividing by $\theta>0$, and passing to the limit $\theta \rightarrow 0$, the following equation is then deduced:

$$
\int_{0}^{l}\left\{E I w^{\prime \prime} z^{\prime \prime}+P w^{\prime} z^{\prime}-f z\right\} d x+\langle\hat{p}, \hat{z}\rangle=0,
$$


i.e.

$$
\int_{0}^{l}\left\{E I w^{\prime \prime} z^{\prime \prime}+P w^{\prime} z^{\prime}+p z-f z\right\} d x+p_{0} z(0)+p_{l} z(l)=0
$$

for every $z \in V$. Since any $z \in C_{0}^{\infty}(0, l)$ is an admissible deflection of the beam, (3.12) implies

$$
\int_{0}^{l}\left\{E I w^{\prime \prime} z^{\prime \prime}+p w^{\prime} z^{\prime}-f z\right\} d x=0, \quad \forall z \in C_{0}^{\infty}(0, l)
$$

This means that

$$
\left(E I w^{\prime \prime}\right)^{\prime \prime}-P w^{\prime \prime}+p=f \text { in }(0, l)
$$

with regard to distribution. If $w$ is smooth enough that $\left(E I w^{\prime \prime}\right)^{\prime}$ and $E I w^{\prime \prime}$ are meaningful at the boundary of $(0, l),(3.12)$ is further reduced to

$$
-\left.\left(E I w^{\prime \prime}\right)^{\prime} z\right|_{0} ^{l}+\left.E I w^{\prime \prime} z^{\prime}\right|_{0} ^{l}+p_{0} z(0)+p_{l} z(l)=0
$$

for every $z \in V$. This precisely implies

$$
\begin{gathered}
\left(E I w^{\prime \prime}\right)^{\prime}(0)+p_{0}=0, \quad-\left(E I w^{\prime \prime}\right)^{\prime}(l)+p_{l}=0, \\
E I w^{\prime \prime}(0)=E I w^{\prime \prime}(l)=0 .
\end{gathered}
$$

Therefore, with the assumption of the smoothness of $w$, it is proved that the saddle point $\{w, \hat{p}\}$ satisfies equilibrium equations (2.11) through (2.13) of the beam.

THEOREM 1. The saddle point $\{w, \hat{p}\} \in V \times K$ of the functional $L(v, \hat{q})$ defined by (3.9) on the set $V \times K$ is a solution of the boundary-value problem $\{(2.3),(2.10)$ through (2.13)\} of the contact problem, whenever $w$ is smooth enough so that $\left(E I w^{\prime \prime}\right)^{\prime}$ and $E I w^{\prime \prime}$ are meaningful at the end points of the beam.

4. An existence theorem. The admissible sets $V$ and $K$ for the deflection of the beam and for the contact pressure of the foundation have not yet been defined. Specification of these follows from investigation of the strain energy of the beam and the total potential energy of the foundation.

Let $E I(x) \in L^{\infty}(0, l)$ and let

$$
m_{1} \leq E I(x) \leq m_{2} \text { almost everywhere in }(0, l)
$$

where $m_{1}$ and $m_{2}$ are positive constants. Then, from (3.8),

$$
F_{w}(v) \leq \max \left(m_{2},|P|\right)\|v\|_{2}^{2}-\int_{0}^{l} f v d x
$$

where

$$
\|v\|_{m}^{2}=\sum_{i \leq m} \int_{0}^{l}\left(d^{i} v / d x^{i}\right)^{2} d x, \quad m \geq 0 .
$$

Here $\|\cdot\|_{m}$ is the norm of the Sobolev space

$$
H^{m}(0, l)=\left\{v: d^{i} v / d x^{i} \in L^{2}(0, l), \quad i=0,1, \ldots, m\right\}
$$


By the Sobolev imbedding theorem and the trace theorem,

$$
H^{2}(0, l) \hookrightarrow C^{1}[0, l]
$$

Here $\hookrightarrow$ means "is densely and continuously embedded," and $C^{m}[0, l]$ is the space of continuously $m$-times differentiable functions defined on the closed interval $[0, l]$. Details of such functional analysis can be found in, e.g., Nečas [5]. Then if $f \in L^{1}(0, l)$,

$$
F_{w}(v) \leq \max \left(m_{2},|P|\right)\|v\|_{2}^{2}+C\|f\|_{0,1}\|v\|_{2}
$$

where $\|\cdot\|_{0,1}$ is the $L^{1}$-norm:

$$
\|f\|_{0,1}=\int_{0}^{l}|f| d x .
$$

The estimate (4.6) means that the potential energy of the beam is finite if $V$ is $H^{2}(0, l)$.

Remark 1. Using (4.5), not only the transverse load $f(x)$ but also the distributed moment $g(x)$, the point load $f_{i}$ at $x=x_{i}, i=1, \ldots, i_{f}$, and the point moment $g_{i}$, at $x=x_{i}, i=1, \ldots, i_{g}$ can be applied on the beam and its end points. Then the term $-\int_{0}^{l} f v d x$ in (3.8) is changed by

$$
-\int_{0}^{l} f v d x-\int_{0}^{l} g v^{\prime} d x-\sum_{i=1}^{i_{f}} f_{i} v\left(x_{i}\right)-\sum_{i=1}^{i_{g}} g_{i} v^{\prime}\left(x_{i}\right) .
$$

This is still meaningful on $H^{2}(0, l)$ if $g \in L^{1}(0, l)$ and $f_{i}, g_{i} \in \mathbb{R}$.

Moreover, (4.5) means that the gradient $\theta=d w / d x$ of the deflection $w \in H^{2}(0, l)$ is continuous on $[0, l]$. This is compatible with physical intuitions, and therefore the Sobolev space $H^{2}(0, l)$ is proper for formulation of the beam problems.

In order to define the admissible set $K$ for contact pressure $\hat{p}(x)$, the differential equation (2.2) (which characterizes the Pasternak foundation) is investigated. The minimum requirement for making sense of (2.2) is the assumption that the admissible set for the deflection of the foundation is $H^{1}(\mathbb{R})$. Indeed, the formal operator of the bilinear form

$$
b(u, v)=\int_{\mathbb{B}}\left\{\mu u^{\prime} v^{\prime}+k u v\right\} d x
$$

is the differential operator

$$
u \rightarrow-\left(\mu u^{\prime}\right)^{\prime}+k u .
$$

The bilinear form $b(u, v)$ makes sense in $H^{1}(\mathbb{R})$, since

$$
b(u, v) \leq \max \left(\mu_{2}, k_{2}\right)\|u\|_{1}\|v\|_{1}
$$

whenever positive constants $\mu_{1}, \mu_{2}, k_{1}$, and $k_{2}$ exist such that

$$
\mu_{1} \leq \mu(x) \leq \mu_{2}, \quad k_{1} \leq k(x) \leq k_{2} .
$$

Moreover, the bilinear form $b(u, v)$ satisfies

$$
b(u, u) \geq \min \left(\mu_{1}, k_{1}\right)\|u\|_{1}^{2} .
$$


Thus, the data $\hat{p}$ in $(2.2)$ refer to the dual space of $H^{1}(\mathbb{R})$; i.e., $\hat{p} \in H^{-1}(\mathbb{R})$. The order $\geq$ in $H^{-1}(\mathbb{R})$ means that

$$
\begin{gathered}
\hat{p} \geq 0 \text { in } H^{-1}(\mathbb{R}) \text { if and only if }\langle\hat{p}, v\rangle \geq 0 \\
\text { for every } v \in H^{1}(\mathbb{R}) \text { such that } v \geq 0 \text { in } \mathbb{R},
\end{gathered}
$$

where $\langle\cdot, \cdot\rangle$ is the duality pairing on $H^{-1}(\mathbb{R}) \times H^{1}(\mathbb{R})$. If $\hat{p} \in L^{2}(\mathbb{R})$, then

$$
\langle\hat{p}, v\rangle=\int_{\mathbb{B}} \hat{p} v d x, \quad \forall v \in H^{1}(\mathbb{R})
$$

It is notable that the Dirac delta function $\delta(x)$ belongs to $H^{-1}(\mathbb{R})$. Therefore the admissible set $K$ for contact pressures can be given by

$$
K=\left\{q \in H^{-1}(\mathbb{R}): q \geq 0\right\} .
$$

Remark 2. As shown in (4.8) and (4.10), the bilinear form $b(u, v)$ is continuous and coercive on $H^{1}(\mathbb{R})$. Then, by the Lax-Milgram theorem (Nečas [5]), for every $\hat{p} \in H^{-1}(\mathbb{R})$, there exists a unique solution $u \in H^{1}(\mathbb{R})$ such that

$$
b(u, v)=\langle\hat{p}, v\rangle, \quad \forall v \in H^{1}(\mathbb{R}) .
$$

This implies the existence of the operator $G$ (which is called Green's operator) from $H^{-1}(\mathbb{R})$ onto $H^{1}(\mathbb{R})$ such that

$$
u=G(\hat{p}) .
$$

Thus, while $\mu$ and $k$ were assumed to be constants in Sec. 2, this is not necessary in order to develop the above theory provided (4.9) holds.

The existence of saddle points $L(v, \hat{q})$ on $V \times K$ follows from Ekeland and Temam's $[1]^{*}$ general existence theorem of saddle points if all requirements are satisfied.

Proposition 1. Let $M$ and $K$ be closed convex subsets of reflexive Banach spaces $V$ and $Y$, respectively. Suppose that $L(v, \hat{q})$ is a functional on $M \times K$ such that

(i) $L(\cdot, \hat{q})$ is convex lower semicontinuous on $M$ for every fixed $\hat{q} \in K$,

(ii) $L(v, \cdot)$ is concave upper semicontinuous on $K$ for every fixed $v \in M$,

(iii) $L(v, \hat{q}) \rightarrow-\infty$ as $\|\hat{q}\|_{Y} \rightarrow+\infty, q \in K$, and

(iv) $\sup _{\hat{q} \in K} L(v, \hat{q}) \rightarrow+\infty$ as $\|v\|_{V} \rightarrow+\infty, v \in M$.

There then exists at least one solution to the saddle point problem:

$$
(w, \hat{p}) \in M \times K: L(w, \hat{q}) \leq L(w, \hat{p}) \leq L(v, \hat{p}),
$$

for every $(v, \hat{q}) \in M \times K$.

Since conditions (i), (ii), and (iii) are obviously satisfied by the Lagrangian $L(v, \hat{q})$ defined by (3.9), it suffices to show that the functional $L(v, \hat{q})$ satisfies condition (iv): coerciveness with respect to $v$.

Let $\hat{q}_{v} \in K$ be a maximizer of $L(v, \hat{q})$ on the set $K$ for a fixed but arbitrary element $v \in V$, i.e.,

$$
L\left(v, \hat{q}_{v}\right)=\sup _{\hat{q} \in K} L(v, \hat{q}) .
$$

\footnotetext{
* The statements of Proposition 1 are properly modified; cf. Ekeland and Temam [1, p. 176].
} 
Then $\hat{q}_{v} \in K$ satisfies the inequality

$$
\left\langle\hat{q}-\hat{q}_{v}, G\left(\hat{q}_{v}\right)-\hat{v}\right\rangle \geq 0, \quad \forall \hat{q} \in K
$$

This is also equivalent to the system

$$
\begin{aligned}
\left\langle\hat{q}, G\left(\hat{q}_{v}\right)-\hat{v}\right\rangle & \geq 0, \quad \forall \hat{q} \in K, \\
\left\langle\hat{q}_{v}, G\left(\hat{q}_{v}\right)-\hat{v}\right\rangle & =0 .
\end{aligned}
$$

The second equation implies

$$
L\left(v, \hat{q}_{v}\right)=\frac{1}{2} \int_{0}^{l}\left\{E I\left(v^{\prime \prime}\right)^{2}+P\left(v^{\prime}\right)^{2}-2 f v\right\} d x+\frac{1}{2}\left\langle\hat{q}_{v}, G\left(\hat{q}_{v}\right)\right\rangle .
$$

It is recalled that the space $V=H^{2}(0, l)$ is decomposed by the sum of two spaces $V \cap \mathscr{R}^{\perp}$ and $\mathscr{R}$, i.e.,

$$
V=V \cap \mathscr{R}^{\perp} \oplus \mathscr{R}
$$

where $\mathscr{R}^{\perp}$ is the orthogonal complement of $\mathscr{R}$ with respect to the space $H^{1}(0, l)$ and $\mathscr{R}$ is defined by the set of all rigid body motions of the beam:

$$
\mathscr{R}=\left\{r \in H^{2}(0, l): r(x)=a x+b, a, b \in \mathbb{R}\right\} .
$$

Then every element $v \in H^{2}(0, l)$ can be decomposed by $v=\tilde{v}+r_{v}, \tilde{v} \in \mathscr{R}^{\perp}$ and $r_{v} \in \mathscr{h}$, where $r_{v}$ is defined by

$$
\left\|r_{v}-v\right\|_{1} \leq\|r-v\|_{1}, \quad \forall r \in \mathscr{R} .
$$

This means that " $\|v\|_{2} \rightarrow+\infty$ " is achieved by " $|\tilde{v}|_{2} \rightarrow+\infty$ " or " $\left\|r_{v}\right\|_{1} \rightarrow+\infty$ ", where $|\cdot|_{2}$ is the seminorm of $H^{2}(0, l)$ defined by

$$
|v|_{2}^{2}=\int_{0}^{l}\left(v^{\prime \prime}\right)^{2} d x
$$

Thus, if (4.1) and $P \geq 0$ hold,

$$
L\left(v, \hat{q}_{v}\right) \geq \frac{1}{2} m_{1}|\tilde{v}|_{2}^{2}-C\|f\|_{0,1}\|v\|_{2} .
$$

Here we have uscd the inequality

$$
\langle\hat{q}, G(\hat{q})\rangle \geq 0, \quad \forall \hat{q} \in H^{-1}(\mathbb{R}) .
$$

Coerciveness of $L\left(\cdot, \hat{q}_{v}\right)$ on $V \cap \mathscr{R}^{\perp}$ follows from the estimate (4.17), i.e., it is determined that $L\left(v, \hat{q}_{v}\right) \rightarrow+\infty$ as $|\tilde{v}|_{2} \rightarrow+\infty$. Thus, in order to show condition (iv), it suffices to show coerciveness of $L\left(\cdot, \hat{q}_{v}\right)$ on $\mathscr{R}$, because of the decomposition (4.15). For every $r \in \mathscr{R}$,

$$
L\left(r, \hat{q}_{r}\right) \geq-a \int_{0}^{l} f(x) x d x-b \int_{0}^{l} f(x) d x+\frac{1}{2}\left\langle\hat{q}_{r}, G\left(\hat{q}_{r}\right)\right\rangle
$$

if $P \geq 0$.

If $r(x)=a x+b>0$ is satisfied on any subset of $[0, l]$ in which measure is positive, there exists a positive constant $c>0$ such that

$$
\left\langle\hat{q}_{r}, G\left(\hat{q}_{r}\right)\right\rangle \geq c\|r\|_{1}^{2}, \quad \hat{q}_{r} \in K .
$$


This is assured by (4.8) and (4.10). Then estimates (4.18) and (4.19) imply

$$
L\left(r, \hat{q}_{r}\right) \rightarrow+\infty \text { as }\|r\|_{1} \rightarrow+\infty,
$$

since the first two terms of (4.18) increase linearly in $\|r\|_{1}$ while the third increases quadratically.

Suppose that $r(x)=a x+b \leq 0$ on $[0, l]$. This implies $G\left(\hat{q}_{r}\right)=0$. Then the estimate (4.19) cannot be applied to show coerciveness of $L\left(r, \hat{q}_{r}\right)$. In this case, other considerations are necessary. Since $a x+b \leq 0$ for every $x \in[0, l]$, then

$$
b \leq 0 \text { and } a \leq-b / l \text {. }
$$

Suppose that

$$
\int_{0}^{l} f(x) d x>0 \text { and } \int_{0}^{l} f(x) x d x>0
$$

Then, from (4.18),

$$
L\left(r, \hat{q}_{r}\right) \geq\left(\frac{1}{l} \int_{0}^{l} f(x) x d x-\int_{0}^{l} f(x) d x\right) b .
$$

This implies that

$$
\left.L\left(r, \hat{q}_{r}\right) \rightarrow+\infty \quad \text { as } \quad b \rightarrow-\infty \quad \text { (i.e. }\|r\|_{1} \rightarrow+\infty\right)
$$

whenever the following condition holds:

$$
\int_{0}^{l} f(x) d x>\frac{1}{l} \int_{0}^{l} f(x) x d x .
$$

If $a \rightarrow+\infty$, then $b \rightarrow-\infty$, using (4.21). Then the same conditions, (4.22) and (4.23), imply that

$$
\left.L\left(r, \hat{q}_{r}\right) \rightarrow+\infty \quad \text { as } \quad a \rightarrow+\infty \quad \text { (i.e. }\|r\|_{1} \rightarrow+\infty\right) .
$$

For $a \rightarrow-\infty$, condition (4.22) implies that

$$
\left.L\left(r, \hat{q}_{r}\right) \rightarrow+\infty \text { as } a \rightarrow-\infty \quad \text { (i.e. }\|r\|_{1} \rightarrow+\infty\right)
$$

Therefore, under conditions (4.22) and (4.23), i.e.

$$
\int_{0}^{l} f(x) d x>\frac{1}{l} \int_{0}^{l} f(x) x d x>0,
$$

coerciveness of $L\left(r, \hat{q}_{r}\right)$ on $\mathscr{R}$ is satisfied. That is,

$$
L\left(r, \hat{q}_{r}\right) \rightarrow+\infty \quad \text { as } \quad\|r\|_{1} \rightarrow+\infty, \quad r \in \mathscr{R}
$$

Thus, from the above discussions, the following theorem can be summarized:

THeorem 2. Let $P \geq 0$. Suppose that condition (4.24), i.e.

$$
\int_{0}^{l} f(x) d x>\frac{1}{l} \int_{0}^{l} f(x) x d x>0,
$$

holds. Then there exists at least one solution, $\{w, \hat{p}\} \in V \times K$, to the saddle-point problem (3.10), i.e.

$$
\{w, \hat{p}\} \in V \times K: L(w, \hat{q}) \leq L(w, \hat{p}) \leq L(v, \hat{p}) \quad \text { for every } \quad\{v, \hat{q}\} \in V \times K .
$$


Remark 3. If, in addition to the transverse load, transverse moment, point loads, and point moments are applied on the beam (i.e., if (4.7) is used instead of $\int_{0}^{l} f v d x$ in (3.8)) condition (4.24) is changed by

$$
\int_{0}^{l} f d x+\sum_{i=1}^{i_{f}} f_{i}>\frac{1}{l}\left(\int_{0}^{l} f x d x+\int_{0}^{l} g d x+\sum_{i=1}^{i_{f}} f_{i} x_{i}+\sum_{i=1}^{i_{g}} g_{i}\right)>0 .
$$

Condition (4.24) or (4.25) is called the compatibility condition and is sufficient for the solution to the contact problem $\{(2.3),(2.10)$ through $(2.13)\}$. Several typical examples are shown in Fig. 2.

It is notable that existence of solutions to the contact problem is not assured even for very small compressible axial forces, $P<0$. In these cases, one of the end points must be fixed or both end points simply supported.

5. An approximation and a solution method. A brief discussion of an approximation of the Lagrangian $L(v, \hat{q})(3.9)$ is first given, and then a solution method for the approximate saddle point problem (3.10) is introduced.

Discretization of the beam and the foundation is performed in such a manner that nodal points of the beam coincide with those of the foundation. Then on each divided element of the beam, the deflection $w(x)$ is interpolated by the Hermite-type cubic polynomials:

$$
w^{e}(x)=\sum_{i=1}^{2} w_{i}^{e} N_{i}^{e}(x)+\theta_{i}^{e} M_{i}^{e}(x) \text { in the eth element, }
$$

where

$$
\begin{array}{ll}
N_{1}^{e}(x)=1-3 x^{2}+2 x^{3}, & M_{1}^{e}(x)=h^{e} x(x-1)^{2} \\
N_{2}^{e}(x)=3 x^{2}-2 x^{3}, & M_{2}^{e}(x)=h^{e} x^{2}(x-1)
\end{array}
$$
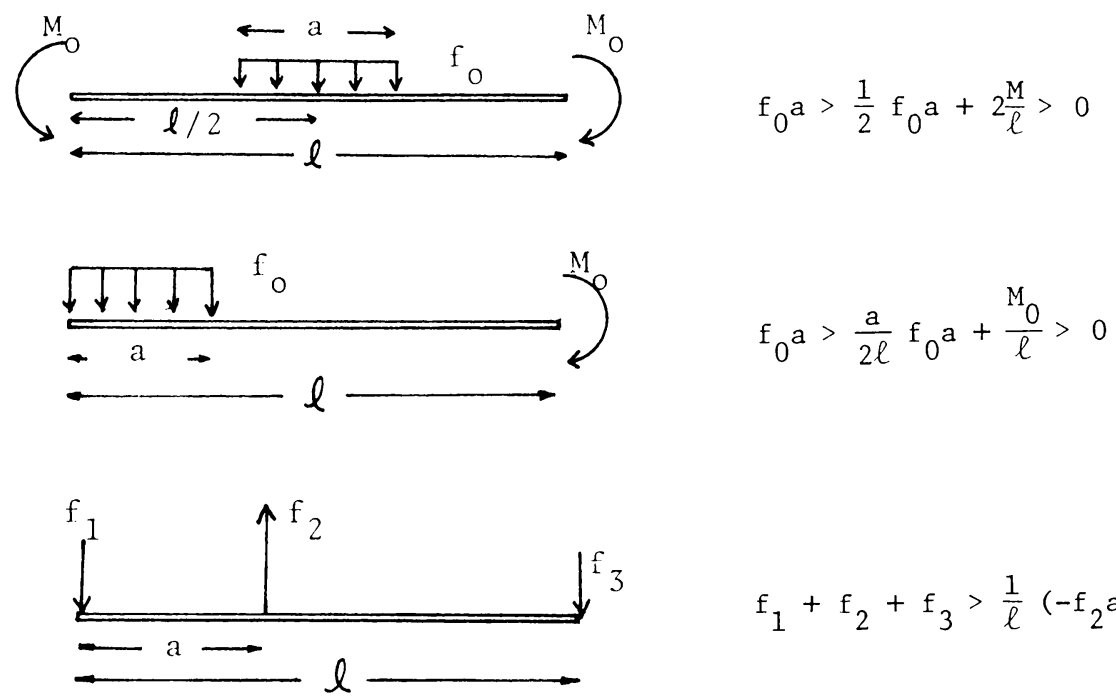

$$
\mathrm{f}_{1}+\mathrm{f}_{2}+\mathrm{f}_{3}>\frac{1}{\ell}\left(-\mathrm{f}_{2} \mathrm{a}+\mathrm{f}_{3} l\right)>0
$$

Fig. 2. Examples of compatible applied forces and moments. 
Here $h^{e}$ is the length of the eth element, and $\left\{w_{i}^{e}\right\}$ and $\left\{\theta_{i}^{e}\right\}$ are the values of the deflection and the gradient of the deflection at the local $i$ th nodal point of the eth element. Assembling each interpolated deflection $w^{e}(x)$, the total deflection of the beam $w(x)$ can be given by

$$
w(x)=\sum_{i=1}^{N} w_{i} N_{i}(x)+\theta_{i} M_{i},
$$

where $N$ is the total number of nodal points of the finite-element model of the beam, and $\left\{N_{i}\right\}_{i=1}^{N}$ and $\left\{M_{i}\right\}_{i=1}^{N}$ are global interpolation functions which are constructed by assembling all $\left\{N_{i}^{e}\right\}_{i=1, e=1}^{2, N-1}$ and $\left\{M_{i}^{e}\right\}_{i=1, e=1}^{2, N-1}$. Substitution of (5.3) into the first three terms of (3.9) then yields

$$
\frac{1}{2} \int_{0}^{l}\left\{E I\left(v^{\prime \prime}\right)^{2}+P\left(v^{\prime}\right)^{2}-2 f v\right\} d x=\frac{1}{2}\left\{V_{i}\right\}^{T}\left[K_{i j}\right]\left\{V_{j}\right\}+\left\{V_{i}\right\}^{T}\left\{F_{i}\right\}, \quad 1 \leq i, j \leq 2 N .
$$

Here, for $1 \leq i, j \leq N, V_{2 i-1}=v_{i}, V_{2 i}=\theta_{i}$, etc.

Next a discretization for the contact pressure $\hat{p}$ is introduced. It is recalled that $\hat{p} \in H^{-1}(\mathbb{R})$ in general. By Sobolev's embedding theorem (see Nečas [5]) the dual space $H^{1}(\mathbb{R})$ of $H^{-1}(\mathbb{R})$ is included in the space $C(\mathbb{R})$ of continuous functions. Then any element $\hat{p} \in H^{-1}(\mathbb{R})$ can be approximated by

$$
\hat{p}(x)=\sum_{i=1}^{M} p_{i} \delta\left(x_{i}\right), \quad p_{i} \geq 0
$$

where $\delta\left(x_{i}\right)$ is the Dirac delta function at the coordinate $x_{i}$, and $p_{i}$ is the value of $\hat{p}$ at $x=x_{i}$. As shown in (2.6), only the portion of the foundation which coincides with the beam should be considered. Then $x_{i}$ is identified with the coordinate of the $i$ th nodal point of the beam, i.e. $M=N$. This identification implies that $p_{1}$ in (5.5) corresponds to $p_{0}$ in (2.6) and that $p_{N}$ in (5.5) corresponds to $p_{l}$ in (2.6). Substitution of (5.5) into (2.3) therefore implies

$$
G(\hat{p})(x)=\sum_{i=1}^{N} g\left(x, y_{i}\right) p_{i}, \quad\left(y_{i}=x_{i}\right)
$$

Then it can be determined that

$$
-\frac{1}{2}\langle\hat{q}, G(\hat{q})\rangle+\langle\hat{q}, \hat{v}\rangle=-\frac{1}{2}\left\{q_{i}\right\}^{T}\left[G_{i j}\right]\left\{q_{j}\right\}+\left\{q_{i}\right\}^{T}\left\{v_{i}\right\}, \quad 1 \leq i, j \leq N
$$

where

$$
G_{i j}=g\left(x_{i}, y_{j}\right) \quad\left(y_{j}=x_{j}\right)
$$

Therefore, the Lagrangian (3.9) can be approximated by

$$
L(\{V\},\{q\})=\frac{1}{2}\left\{V_{i}\right\}^{T}\left[K_{i j}\right]\left\{V_{j}\right\}+\left\{V_{i}\right\}^{T}\left\{F_{i}\right\}-\frac{1}{2}\left\{q_{i}\right\}^{T}\left[G_{i j}\right]\left\{q_{j}\right\}+\left\{q_{i}\right\}^{T}\left\{v_{i}\right\} .
$$

The discretized saddle point $\{\{W\},\{p\}\}$ of the Lagrangian $L(\{V\},\{q\})$, i.e. the solution of the discretization of the saddle-point problem (3.10), can be characterized by

$$
\begin{aligned}
& \left\{p_{j}\right\} \geq 0:\left(\left\{q_{i}\right\}-\left\{p_{i}\right\}\right)^{T}\left(\left[G_{i j}\right]\left\{p_{j}\right\}-\left\{w_{i}\right\}\right) \geq 0 \text { for every }\left\{q_{i}\right\} \geq 0, \\
& \left\{W_{j}\right\}:\left\{V_{i}\right\}^{T}\left(\left[K_{i j}\right]\left\{W_{j}\right\}-\left\{F_{i}\right\}\right)+\left\{v_{i}\right\}^{T}\left\{p_{i}\right\}=0 \quad \text { for every }\left\{V_{i}\right\},
\end{aligned}
$$

as in (3.11) and (3.12). That is, the coupled system (5.10) and (5.11) has been obtained. 
The solution $\left\{\left\{p_{j}\right\},\left\{w_{j}\right\}\right\}$ of the coupled system (5.10) and (5.11) is then obtained by using the following simple problem:

$$
x \geq 0:(y-x)(x-z) \geq 0, \quad \forall y \geq 0
$$

where $x, y$ and $z$ are real numbers. The solution $x$ of (5.12) is

$$
x=\operatorname{Max}(0, z) \text {. }
$$

Indeed, if $z \geq 0$,

$$
(y-\operatorname{Max}(0, z))(\operatorname{Max}(0, z)-z)=0 .
$$

If $z<0$,

$$
(y-\operatorname{Max}(0, z))(\operatorname{Max}(0, z)-z)=y(-z) \geq 0, \quad \forall y \geq 0 .
$$

Moreover, the solution of (5.12) is unique. Suppose that $x_{1} \geq 0$ and $x_{2} \geq 0$ are solutions of (5.12). Then

$$
\left(x_{2}-x_{1}\right)\left(x_{1}-z\right) \geq 0, \quad\left(x_{1}-x_{2}\right)\left(x_{2}-z\right) \geq 0 .
$$

The addition of the two inequalities implies

$$
\left(x_{1}-x_{2}\right)^{2} \leq 0, \quad \text { i.e. } \quad x_{1}=x_{2} .
$$

Therefore, (5.13) offers a unique solution to inequality (5.12).

The above solution for $\mathbb{R}$ may also be extended to $\mathbb{R}^{N}$; i.e., the solution of

$$
\left\{x_{i}\right\} \geq 0:\left(\left\{y_{i}\right\}-\left\{x_{i}\right\}\right)^{T}\left(\left\{x_{i}\right\}-\left\{z_{i}\right\}\right) \geq 0, \quad \forall\left\{y_{i}\right\} \geq 0
$$

can be given by

$$
\left\{x_{i}\right\}=\operatorname{Max}\left(\{0\},\left\{z_{i}\right\}\right) .
$$

Thus, in conclusion, the solution of (5.10) can be written as

$$
\left\{p_{i}\right\}=\operatorname{Max}\left(\{0\}, \quad\left\{p_{i}\right\}-\rho\left(\left[G_{i j}\right]\left\{p_{j}\right\}-\left\{w_{i}\right\}\right)\right), \quad \rho>0,
$$

where $\rho$ is an arbitrary positive number.

Conversely, if the solution of (5.14) is obtained for some $\rho>0$, it will also apply to inequality (5.10). Furthermore, the form of (5.14) suggests that the solution may be obtained by the iterative contraction procedure. Thus, the following iterative method, after Uzawa's scheme (Ekeland and Temam [1]), is proposed to obtain the solution of (5.10) and (5.11):

(i) Assume the initial contact pressure

$$
\left\{p_{i}^{1}\right\}, \quad p_{i}^{1} \geq 0 .
$$

(ii) For the $t$ th iteration solve the problem

$$
\left\{W_{j}^{t+1}\right\}:\left\{V_{i}\right\}^{T}\left(\left[K_{i j}\right]\left\{W_{j}^{t+1}\right\}-\left\{F_{i}\right\}\right)+\left\{v_{i}\right\}^{T}\left\{p_{i}^{t}\right\}=0 .
$$

(iii) Obtain the $(t+1)$ th contact pressure through

$$
p_{i}^{t+1}=\operatorname{Max}\left(0, p_{i}^{t}-\rho\left(\sum_{j=1}^{i-1} G_{i j} p_{j}^{t+1}+\sum_{j=1}^{N} G_{i j} p_{j}^{t}-w_{i}^{t+1}\right)\right) .
$$

(iv) Check the convergence of $\left\{W_{j}^{t+1}\right\}$ and $\left\{p_{i}^{t+1}\right\}$. 
One method of finding the initial contact pressure $\left\{p_{i}^{1}\right\}$ is to solve the bilateral problem and obtain the contribution of the foundation. Here "bilateral" means that the beam is connected with the foundation. Neglecting the negative pressures obtained by the bilateral problem, $\left\{p_{i}^{1}\right\}$ is obtained.

If

$$
\rho=\omega / G_{i i}
$$

in (5.17) is chosen, the iteration form (5.17) becomes the projectional S.O.R. method.

Also, in the iterative procedure the stiffness matrix $\left[K_{i j}\right]$ is not invertible unless fixed or supported conditions of the beam are imposed; that is, (5.16) may not be easily solved by taking the inverse of $\left[K_{i j}\right]$. To overcome this difficulty when no supported or fixed conditions are imposed, two additional conditions are implied from (variational) equation (3.12). Since (3.12) holds for any $z \in V=H^{2}(0, l),(3.12)$ is satisfied for

$$
z=a x+b, \quad a, b \in \mathbb{R}
$$

i.e.,

$$
\left.a_{\{} P(w(l)-w(0))+\int_{0}^{l} p x d x+p_{l} l-\int_{0}^{l} f x d x\right\}+b\left\{\int_{0}^{l} p d x+p_{0}+p_{l}-\int_{0}^{l} f d x\right\}=0
$$

for every $a, b \in \mathbb{R}$. This implies

$$
\begin{gathered}
P(w(l)-w(0))+\int_{0}^{l} p x d x+p_{l} l-\int_{0}^{l} f x d x=0, \\
\int_{0}^{l} p d x+p_{0}+p_{l}-\int_{0}^{l} f d x=0 .
\end{gathered}
$$

The first equation of (5.19) signifies the balance of moments around the origin, and the second signifies the balance of forces in the transverse direction. The discretized version of $(5.19)$ is given by

$$
\begin{gathered}
P\left(w_{N}-w_{1}\right)+\sum_{i=1}^{N} p_{i} x_{i}-\sum_{i=1}^{N} F_{2 i-1} x_{i}=0, \\
\sum_{i=1}^{N} p_{i}-\sum_{i=1}^{N} F_{2 i-1}=0 .
\end{gathered}
$$

Thus, if there are no support or fixed conditions on the beam, Eq. (5.16) is solved together with the conditions of (5.20).

Numerical example. Finally, an example problem of beam bending on a Pasternak foundation is solved using the numerical method discussed above.

Let $l=E I=\mu=k=1.0$. The beam is divided into ten equi-length finite elements, and the foundation is discretized by equi-length elements. Nodal points of the beam coincide with those of the foundation. A point load $f_{0}$ and a point moment $M_{0}$ are applied on the center of the beam. The compatibility condition (4.24) is then satisfied if $f_{0}>\left(f_{0} l / 2+M_{0}\right) / l>0$, i.e.

$$
\frac{1}{2} f_{0} l>M_{0}>-\frac{1}{2} f_{0} l
$$


Suppose that $f_{0}=1.0$ is fixed. Then $(5.21)$ becomes $0.5>M_{0}>-0.5$. Four cases, $M_{0}=0.1, M_{0}=0.3, M_{0}=0.4$ and $M_{0}=0.49$ are solved.

In this particular example, two nodal points from the right end of the beam are assumed to contact the foundation. Taking the initial estimate $\left\{p_{i}^{1}\right\}$ of the contact pressure, which roughly satisfies condition (5.20), the deflection of the foundation is calculated at the two right end points; i.e.,

$$
u_{i}^{1}=G_{i j} P_{j}^{1}, \quad i=10 \text { and } 11
$$

Since the two right end points are assumed to contact the foundation, deflection of the beam can be specified at these points. That is, Eq. (5.16) is solved under the condition $w_{i}^{1}=u_{i}^{1}, i=10$ and 11 , by taking the inverse of $\left[K_{i j}\right]$. Applying (5.17), $\left\{p_{i}^{2}\right\}, i=1, \ldots, 9$, are obtained. The values $p_{10}^{2}$ and $p_{11}^{2}$ are given by the equations

$$
\sum_{i=1}^{9} p_{i}^{2}\left(x_{11}-x_{i}\right)+p_{10}^{2}\left(x_{11}-x_{10}\right)=f_{0}\left(x_{11}-l / 2\right)+M_{0}, \quad p_{11}^{2}=f_{0}-\sum_{i=1}^{10} p_{i}^{2} .
$$

These equations are essentially the same as (5.20). Repeating the above procedures, Uzawa's scheme [(5.15) through (5.17)] converges for sufficiently small $\rho>0$ in (5.17).

Numerical results are shown in Figs. 3(a) through 3(d).

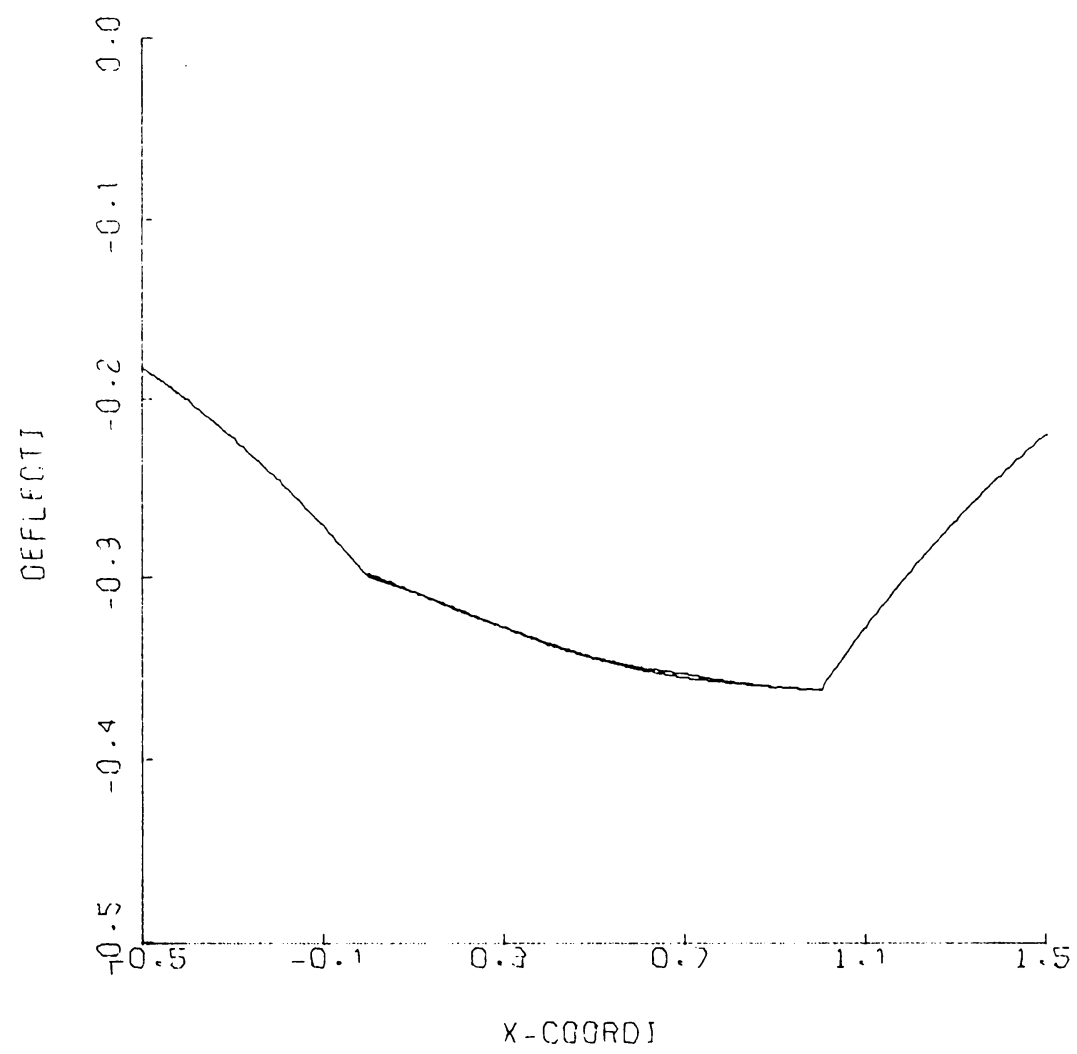

FIG. 3(a). Numerical example-case $\mathbf{I}-(f=-1$ and $M=0.1)$. 

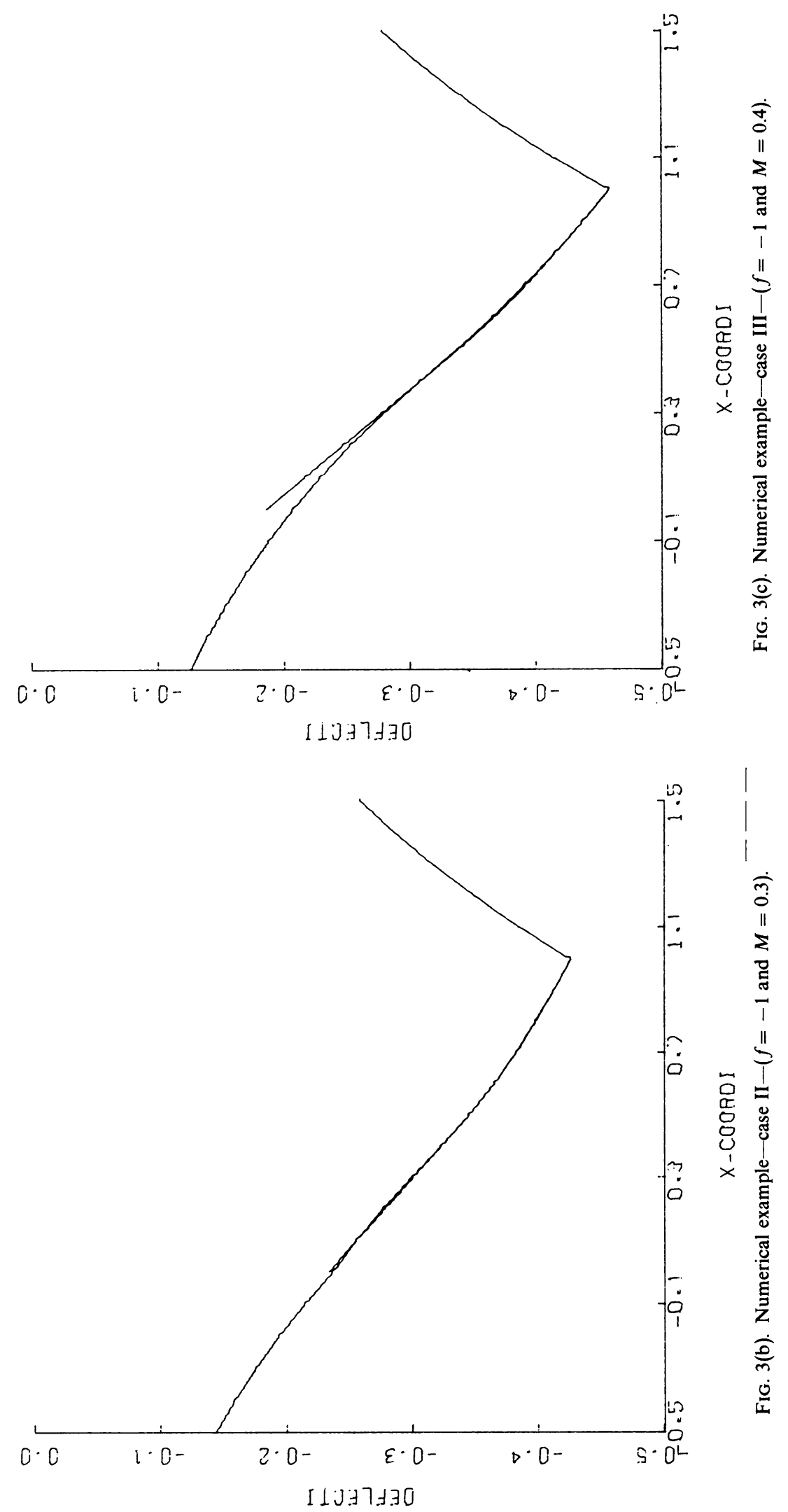


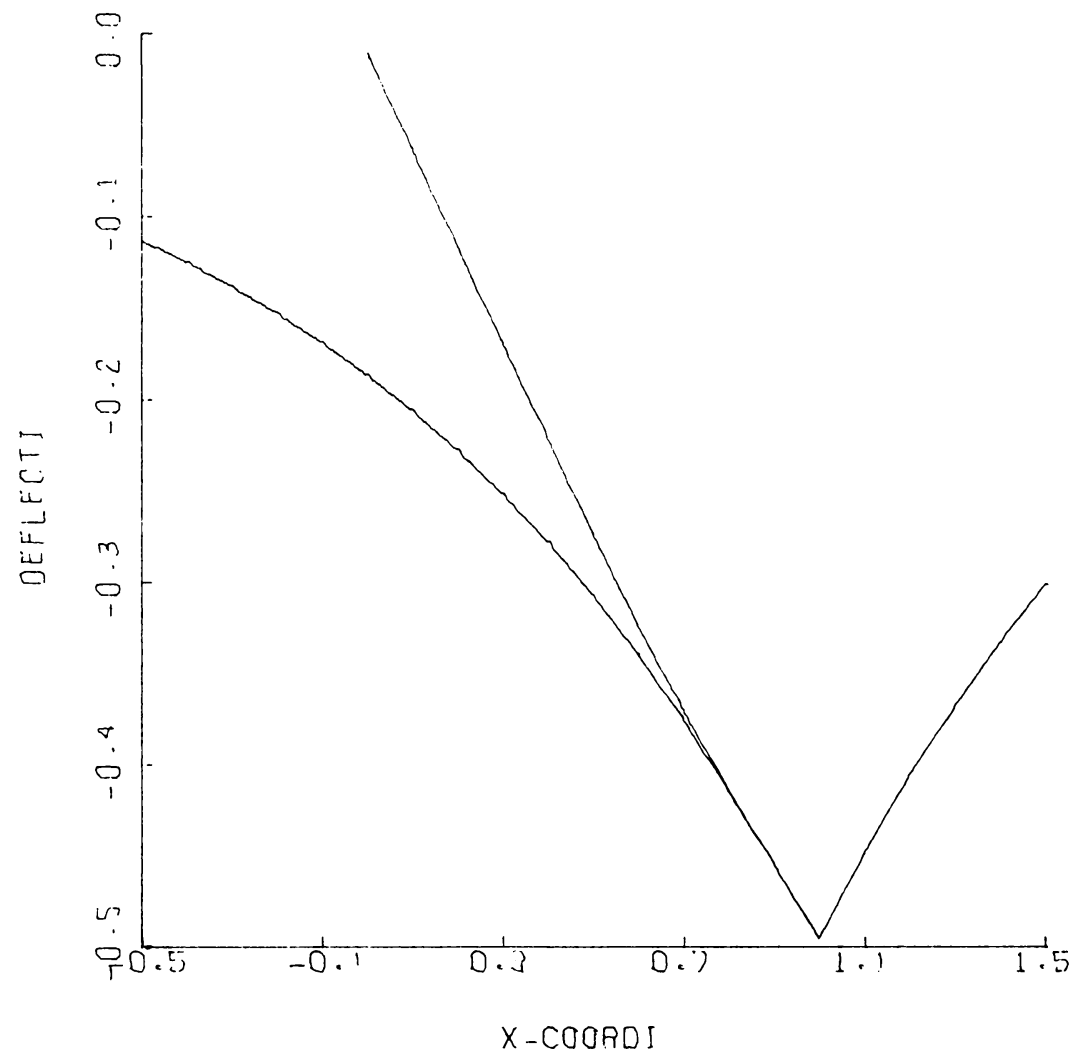

FIG. 3(d). Numerical example - case IV $-(f=-1$ and $M=0.49)$.

\section{REFERENCES}

[1] I. Ekeland and R. Temam, Convex analysis and variational problems, North-Holland, American Elsevier, New York, 1976

[2] M. Fremond, Solid resting on a stratified medium, in Variational methods in engineering II, University of Southampton, 1972 , pp. 8/80-8/86

[3] M. Boucher, Signorini's problem in viscoelasticity, in The mechanics of the contact between deformable bodies, ed. Pater and Kalker, Delft University Press, 1975, p. 41-53

[4] Y. C. Fung, Foundations of solid mechanics, Prentice-Hall, 1975

[5] J. Nečas, Les méthodes directes en theorie de equations elliptiques, Masson, Paris, 1967

[6] A. C. Keer, Elastic and viscoelastic foundation models, J. of Appl. Mech., ASME, 491-498 (1964) 